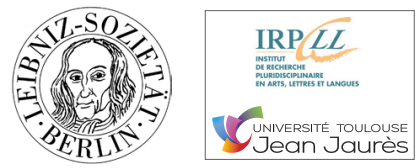

\title{
Etudes culturelles, Cultural Studies, Kulturwissenschaften. Eine vergleichende Skizze im Prisma der Norm
}

\section{Anne Chalard-Fillaudeau}

Université Paris 8 Vincennes-Saint-Denis

\section{Abstract}

Der vorliegende Beitrag stellt einen Versuch dar, anhand eines heuristischen Hilfsmittels, nämlich der Norm, ein signifikantes Ensemble von Gemeinsamkeiten und Unterschieden zwischen Cultural Studies, Kulturwissenschaften, études culturelles zu erfassen und so das aufklärerische Potenzial dieser facettenreichen Konstellation zu beleuchten. Da angesichts der Pluralität dieser wissenschaftlichen Formationen ein ausführlicher Vergleich kaum möglich ist, erscheint es folgerichtig, darüber zu reflektieren, wie sie zum Phänomen Norm stehen und dabei eine Orientierungsfunktion erfüllen, die bestimmte Verfahren voraussetzt und Fragen der Legitimität aufruft.

La présente contribution entend ressaisir un ensemble signifiant d'éléments qui unissent et différencient les approches du culturel dans les aires anglo-saxonnes, germaniques et françaises en envisageant leurs démarches scientifiques respectives au prisme de la norme. Une telle contribution ne saurait en effet prétendre à l'exhaustivité compte tenu de l'extrême pluralité des formations embrassées, mais n'en est pas moins en mesure de pointer ce que ces formations ont de commun et de divers dans leur aspiration à éclairer et donc orienter, dans leurs partis pris théoriques et méthodologiques et dans leur positionnement au sein du paysage scientifique et universitaire.

This paper aims at bringing out some of the most significant characteristics that unite and differentiate the way culture is dealt with in the Cultural Studies, Kulturwissenschaften and études culturelles and does so in the light of norm. It would be indeed unavailing to be fully exhaustive given the great plurality of all this scientific formations. It is certainly more relevant to point out how these formations respectively navigate in relation to the norm, whether in theory and practice or at the institutional level, and in so doing fulfill a crucial orientation function.

\section{Keywords}

Cultural Studies $•$ Kulturwissenschaften $\bullet$ études culturelles $\bullet$ Vergleich $\bullet$ Norm $\bullet$ Interdisziplinarität

Cultural Studies $•$ Kulturwissenschaften $\bullet$ études culturelles $\bullet$ comparaison $\bullet$ norme $\bullet$ interdisciplinarité

Cultural Studies $•$ Kulturwissenschaften • études culturelles $\bullet$ comparison $\bullet$ norm $\bullet$ interdisciplinarity

Dieser Beitrag zu den (inter-)nationalen Varianten der Kulturstudien muss mit einem paradoxen wie auch logischen Befund beginnen: Cultural Studies, Kulturwissenschaften, études culturelles als plastische Konstellationen der englisch-, deutsch- und französischsprachigen Kulturstudien stehen im janusköpfigen Zeichen der Einheitlichkeit Differenziertheit, das heißt: Sie stimmen im Grundsatz überein, weichen aber praktisch und politisch voneinander ab, insofern als sie alle Kultur erforschen [= Einheitlichkeit des Subjektes] und dabei kultur- und kontextbedingt sind [= Differenziertheit der Objekte und Verfahrensweisen]. Sie sind alle in der Kultur verankert [= Einheitlichkeit des Ansatzpunktes] und münden, je nach dem Produktionsort, in verschiedene diskursive Formationen im Sinne der Diskurstheorie von Foucault [= Differenziertheit des
Wissenszusammenhanges und des Erwartungshorizontes], eben deshalb, weil sie eminent kulturell sind und somit auf die (politisch-)wissenschaftliche Agenda antworten.

Pointiert formuliert sind Kulturstudien in ihrer Ähnlichkeit und Vielfalt eine Fallstudie an sich in Bezug auf die Archäologie des Wissens, eine Fallstudie, die umso relevanter und schlüssiger erscheint, als sie unter anderem die Frage der Norm aufwirft - eine überaus aktuelle, kulturelle Frage in einer Welt, die überwiegend nach Orientierung fragt. Kulturstudien mögen zwar ein wenig normal sein in Sachen Kulturanalyse und Interdisziplinarität (normal im Sinne von etwas, das über längere Zeiträume ähnlich ablaufenden Ereignissen entspricht). Sie wirken somit nicht mehr sehr ,originell'; aber sie gründen auf keiner Norm in Sachen Fragestellungen und Verfahrensweisen; wollen auch nicht 
normieren, wenngleich sie in Sachen Offenheit und Interdisziplinarität gewissermaßen normativ sind. Zu guter Letzt: Sie sind nicht völlig normalisiert in Sachen institutioneller Verankerung.

Die Norm stellt daher als ein heuristisches Element eine interessante Facette dar, um sich dem Verhältnis zwischen Cultural Studies, Kulturwissenschaften und études culturelles zu nähern und ihre Identität perspektivisch zu befragen. Dies kann durch eine Positivdefinition geschehen: Sie setzen sich alle drei als Forschungsansätze und -gebiete mit der Norm (als einem strukturierenden Bestandteil der Praktiken und als einem erkenntnistheoretischen Prinzip der Kultur) auseinander. In einer Negativdefinition kann festgestellt werden: Sie sind dennoch keine ganz normalen Formationen in der wissenschaftlichen Landschaft. Hinzu kommt, dass die Art und Weise, wie sie sich mit der Norm bzw. der Kultur auseinandersetzen (können und dürfen), auf den jeweiligen nationalen und wissenschaftlichen Kontext verweist, wodurch Identität mit Heterogenität verbunden sind. Im Prisma der Norm kann man also Cultural Studies, Kulturwissenschaften, études culturelles vergleichen und dabei erstens die Einheitlichkeit herausstellen: Kulturstudien haben eine Orientierungsfunktion, aber übernehmen dabei keine Normierungsfunktion. Sie bahnen vielmehr Wege der Interpretation an. Dann werden wir uns im zweiten Teil mit der Einheitlichkeit-Differenziertheit in der Praxis befassen: Kulturstudien lassen sich unter keine monodisziplinäre Norm der Untersuchung subsumieren und generieren mithin ein vielfältiges Spektrum von Herangehensweisen. Insofern bringen sie eine bestimmte Normativität im Hinblick auf Interdisziplinarität und wissenschaftliche Offenheit hervor. Schließlich kann die Einheitlichkeit-Differenziertheit auch im Hinblick auf den Grad der institutionellen Anerkennung untersucht werden: Kulturstudien haben sich überall ausgebreitet, aber sie sind, je nach Ort, mehr oder weniger normalisiert bzw. normalisierbar.

\section{EINHEITLICHKEIT UND DIFFERENZIERTHEIT AUF DER EBENE DER ORIENTIERUNGSFUNKTION}

Um dem Leser die Orientierung zu erleichtern, soll hier kurz auf die Terminologie eingegangen werden. Wir haben in der Einleitung auf Foucaultsche Begriffe rekurriert, nämlich auf diskursive Formationen und eine Archäologie des Wissens. Uns geht es bei dem Vergleich der Kulturstudien darum, die folgenden Fragen zu beantworten, mit denen sich Foucault intensiv beschäftigt hat: Wie und warum werden in einer bestimmten historischen Epoche bzw. diskursiven Formation bestimmte Äußerungen mit einem bestimmten Sinn verknüpft bzw. wie und warum übernehmen sie eine bestimmte

1 Da es im Rahmen dieses Beitrages nicht möglich ist, einen ausführlichen Vergleich auszuarbeiten, der alle denkbaren Aspekte erfassen würde. Siehe weiterführend dazu: Chalard-Fillaudeau 2015.
Funktion innerhalb des Diskurses, in unserem Fall also eine Orientierungsfunktion.

Außerdem haben wir vereinfachend von études culturelles gesprochen. Denn in den Geistes- und Sozialwissenschaften konnte bislang keine speziell französische Variante und/oder Tradition der Cultural Studies oder Kulturwissenschaften in Form eines Konzeptes, das dem anglo-amerikanischen bzw. deutsch-österreichischen vergleichbar wäre, ausgemacht werden. Heute kann man aber von Präsenz und progressiver Akzeptanz der interdisziplinären Dynamik der Kulturanalyse reden. Mit anderen Worten werden jetzt Cultural Studies und, in geringerem Maße, Kulturwissenschaften rezipiert und es entstehen französische Kulturstudien eigener Art. Hier haben wir es allerdings mit einem ausdifferenzierten Forschungsbereich zu tun, der sich zur eigenständigen transdisziplinären Forschungstradition entwickelt. Diese Entwicklung geht mit einer terminologischen Debatte einher (Darras 2007). In manchen Fällen wird von Cultural Studies gesprochen oder Cultural Studies werden explizit oder implizit durch études culturelles übersetzt. In anderen Fällen aber wird von études culturelles ohne bestimmten Bezug auf Cultural Studies gesprochen. In weiteren Fällen wird von sciences de la culture mit explizitem Bezug auf Kulturwissenschaften gesprochen. Schließlich gibt es noch Initiativen, die analyse culturelle genannt werden und andere, die nicht speziell bezeichnet werden, dafür aber eine Verwandtschaft mit Cultural Studies oder Kulturwissenschaften anzeigen. Jene Vielfalt weist also darauf hin, dass sich études culturelles nicht absolut bzw. nur bedingt mit Cultural Studies oder Kulturwissenschaften decken (siehe Teil II). Andererseits ist zu vermuten, dass es sich um eine Übergangsphase handelt, die mittelfristig auf eine vereinheitlichende Benennung hinauslaufen könnte. Insofern sprechen wir im Folgenden von der immer üblicheren Benennung études culturelles und stellen diese wachsende Formation den angloamerikanischen Cultural Studies und den deutschsprachigen Kulturwissenschaften komparativ zur Seite.

Terminologisch bzw. wissenschaftstheoretisch berufen sich alle drei Formationen auf den diffusen Begriff Kultur. Sie begreifen sich alle drei als Ort der Auseinandersetzung mit Kultur als einer Gesamtheit von Wahrnehmungsschemata, materiellen Produktionen und Äußerungsformen, Praktiken, Prozessen, Strukturen, die Sinn- und Bedeutungszusammenhänge wie auch Handlungszusammenhänge schaffen und veranschaulichen. Angesichts der reichhaltigen Begrifflichkeit von Kultur können wir in dem vorliegenden kurzen Beitrag all die begrifflichen Spezifikationen innerhalb der jeweiligen Forschungskontexte nicht ansprechen, aber doch erklären, dass Kulturstudien allesamt von einer pluralen Auffassung ausgehen, die nach den Organisations- und Ausdrucksformen menschlicher Welterfahrung fragt und dabei die herkömmlichen Wertkategorien neutralisiert, und insbesondere den Gegensatz Hochkultur / Populärkultur einebnet. Pluralität bedeutet hier vor allem auch Akzeptanz des Fremden und des 
Anderen, gehe es um die Themenfelder, Fragestellungen, Theorien oder auch Methoden.

Es ist weiterhin festzuhalten, dass eine solche Auseinandersetzung mit Kultur Antworten auf den Wandel der Kulturen bzw. auf die Herausforderungen, die sich aus dem Wandel ergeben, (wie etwa im Zuge der Bürgerrechtsbewegung in den USA oder des Feminismus und Postkolonialismus in der weiten Welt) anbieten. Dies gilt auch für die Fragen, in denen sich Probleme der Welt(un)ordnung bündeln: wie etwa die der Knappheit von Gütern, Mitteln und Ressourcen und deren asymmetrischer Verteilung (auf deutschsprachigem Gebiet siehe die Zeitschrift für Kulturwissenschaften, die nach der Finanzkrise 2008 nicht nur für die Integration der ökonomischen Fragen in die kulturwissenschaftlichen Projekte, sondern auch für die Integration der ökonomischen Wissensbestände als kulturwissenschaftliche Forschungsobjekte plädierte) - oder die des Multikulturalismus in Frankreich. Dies erklärt nicht nur, warum Kulturstudien zu verschiedenen Zeitpunkten an verschiedenen Forschungsorten in Europa und Amerika entstanden sind - man denke an die gesellschaftlichen Umstrukturierungen in Großbritannien nach dem Zweiten Weltkrieg, an die Ansprüche der amerikanischen Area Studies in den sechziger und siebziger Jahren, an die wissenschaftstheoretische Abweichung von den Geisteswissenschaften in Deutschland in den siebziger und achtziger Jahren, an die heutigen Integrationsmängel in Frankreich. ${ }^{2}$ Diese breiten sich in der Welt weiter aus. Es lassen sich darüber hinaus zwei Dinge feststellen: Kulturstudien haben sich in mannigfacher Gestalt entwickelt, sie streben dabei aber alle nach Wissensvermittlung wie auch Öffnung von Handlungsmöglichkeiten, d.h.: Sie generieren ein praxisrelevantes Wissen, das die Rezipienten zu Akteuren macht, bei dem Weltverständnis und Weltaneignung miteinander verschmelzen.

In dieser Hinsicht sind sie grundsätzlich durch die historische, kulturelle Bedingtheit der Untersuchungen und Aussagen gleichsam normiert und profilieren sich folgerichtig auf differenzierte Weise hinsichtlich der Orientierungsfunktion. Die jeweiligen Genealogien und Zwecke der Cultural Studies und der Kulturwissenschaften sind in vielerlei Hinsicht und vielerorts diskutiert worden (Assmann; Chalard-Fillaudeau; During; Musner, usw.). Im Zusammenhang mit dem Wandel, der die Geistes- und Sozialwissenschaften in eine crisis of humanities stürzte, stehen Cultural Studies im Zeichen des Empowerment, intendieren doch, wie Hall und Grossberg es so hartnäckig vertreten haben, Menschen die intellektuellen Ressourcen zu vermitteln, die es ihnen ermöglichen, angesichts des gesellschaftlich-politischen und ökonomischen Kontextes eigene Überlebens- und/oder Widerstandsstrategien zu erfinden. Im Zusammenhang des Wandels erschließen Kulturwissenschaften wiederum neue Gebiete der Organisations- und Ausdrucksformen menschlicher

2 Dabei sei auf die Anforderungen der Kinder mit Migrationshintergrund verwiesen, die seit dem Anfang der 80 er Jahre bemängeln, dass sie nicht genug sichtbar' sind und dass ihre Zugehörigkeit zur französischen Gesellschaft hinterfragt wird.
Welterfahrung, verschaffen Menschen den Zugang zu den intellektuellen Ressourcen, die es innen ermöglichen, mit dem Wandel umzugehen (mit eher aufklärerischem und nicht so sehr politischem Schwerpunkt) und widmen der Neuorientierung traditioneller Fachbereiche und Fakultäten eine beachtliche Reformenergie (insofern kann man von Wissenschaftspolitik sprechen). Lutz Musner fasst die verschiedenen kulturwissenschaftlichen Intentionen auf folgende Weise zusammen und bezieht sich dabei auf die Norm:

According to such approaches, the function of Kulturwissenschaften ist not to inject sense and normative orientation into the normative vacuum of modern, contingent and relativist societies but rather to strengthen the capacity for reflection and self-reflection, and hence to counterbalance tendencies towards any expressions of political, religious and cultural fundamentalism.

Thus, the Kulturwissenschaften display themselves often - with the exception of some of their gender studies' variations - as a basically apolitical endeavour which does not seek to empower the social and cultural disadvantage but to set up intellectual barriers against counter-enlightenment and the disastrous strategies of politicizing society at large. Thus the Kulturwissenschaften should not be misinterpreted as ,cultural studies in the Anglo-American sense of the term. (Musner 1999: 580)

Genealogie und Zwecke der études culturelles sind hingegen weniger bekannt. Und doch verfügte Frankreich schon früh über eine kulturwissenschaftliche Matrix. Von Frankreichs Geschichtsforschung (Ecole des Annales), Anthropologie (Lévi-Strauss), Philosophie (French Theory), Semiotik (Barthes) und Soziologie (Bourdieu) gingen in der Tat wichtige Impulse aus. Zudem waren kulturwissenschaftliche Fragestellungen keine Seltenheit in den sechziger und siebziger Jahren: Man denke etwa an Überlegungen zur Massenkultur in der 1962 gegründeten Zeitschrift Communication, mit Edgar Morin als Chefredakteur, Christian Metz (Filmwissenschaft) und Roland Barthes (Semiotik). Dennoch hat die French Theory, die in anderen Ländern mit der Entwicklung der zeitgenössischen bzw. postmodernen Kulturstudien assoziiert ist, in einer Kulturanalyse gleich der Cultural Studies und Kulturwissenschaften keinen direkten Niederschlag gefunden, und das damalige innovative Denken hat zu keiner Umbenennung und Neuorientierung traditioneller Fachbereiche und Fakultäten Anstoß gegeben, auch nicht zur Aufstellung einer politischen Agenda. Die Gründe sind kontext- und kulturbedingt und können hier nur gestreift werden: Bedeutung der Kultur / Kult der Disziplin, Vorrangstellung der Hochkultur, Dynamik der Geschichtswissenschaft bzw. der Kulturgeschichte, Signifikanz des republikanischen Modells, Antiamerikanismus, wissenschaftlicher Parochialismus, Ablösung der Denker der French Theory durch eine neue Generation von französischen Intellektuellen in den achtziger Jahren usw. (Chalard-Fillaudeau 2015). Heute entsteht 
immerhin eine Formation, die über die Fächer hinweg und trotz / wegen des republikanischen Modells die epistemischen und (wissenschafts-)politischen Herausforderungen, gar Schwerfälligkeiten, bewältigen will wie auch die soziokulturellen Herausforderungen. Dieses vibrierende Streben impliziert einen neuen Methoden-Diskurs, dem im Folgenden unser Interesse gilt.

\section{EINHEITLICHKEIT UND DIFFERENZIERTHEIT AUF DER EBENE DER VERFAHREN}

Trotz aller Heterogenität in Bezug auf Zweck und Genealogie bzw. Orientierungsfunktion lassen sich Prinzipien und methodologische Grundsätze artikulieren, die für gute qualitative Forschung bürgen. Denn es geht den Forschungskonstellationen Cultural Studies, Kulturwissenschaften und études culturelles wie jeder traditionellen (normalen?) Forschungstradition um Verantwortung und Zuverlässigkeit: Es ist sozusagen die Norm ihres Handelns. Diese gemeinsame wissenschaftsethische Norm entspricht aber dabei keiner theoretischen und methodologischen Norm, bis auf die primäre Norm des Interdisziplinären oder, allgemeiner gesagt, der pragmatischen Herausbildung eines Denksystems, in dem wir unsere plastische Lebenswelt beschreiben, interpretieren und gar verändern können. Und zwar determinieren die Kulturstudien das Was und Wie des Erforschens nicht präskriptiv, sondern generieren es kreativ: Die Gegenstände konstituieren sich je nach dem Kontext und der Einsicht des Forschers. Es dürfte keine Norm geben, weil die Kontexte variieren und der Umgang individuelle Sache ist, in manchen Fällen gar ,Teamsache' ist (zu jeder Fragestellung ihre Forschungsgruppe...). Pointiert gesagt ist Zuverlässigkeit die wissenschaftsethische Norm der Kulturstudien und die A-Normalität, die dem individuellen Bewusstsein und der subjektiven Intentionalität innewohnt, deren erkenntnistheoretische Norm. Damit sei nicht gesagt, dass man bei dem rein Subjektiven und Einmaligen bleibt, sondern, dass man vom Ich ausgeht, um der individuellen kulturellen Dynamik der Weltaneignung gerecht zu werden und die Konstruktivität der Organisations- und Ausdrucksformen zu reflektieren. (Die Wissenschaftlichkeit der Ergebnisse mag dann im Prisma der Intersubjektivität oder der interdiskursiven kulturwissenschaftlichen Konfigurationen unter Beweis gestellt werden). Cultural Studies, Kulturwissenschaften und études culturelles grenzten sich jeweils von den Geisteswissenschaften und sciences de l'homme ab, um ausgerechnet den Fokus von dem menschlichen Faktor und dem Weltverständnis zu den Strukturen, Prozessen, Praktiken und dem ,Welterlebnis' zu verlagern.

In diesem Zusammenhang lauten die eigentlichen einheitlichen Hauptmerkmale der Untersuchung: Offenheit des Forschungsprozesses, pragmatische Theorien- und Methodenwahl, Reflexivität von Gegenstand und Analyse im Laufe des Forschungsprozesses. Das Prinzip der Offenheit setzt tatsächlich voraus, dass Theorien, Konzepte und Methoden im Zusammenhang mit der Forschungsproblematik entlehnt oder entwickelt, auf jeden Fall zweckmäßig bestimmt werden. Daher kommt es, dass Kulturstudien sich jeder disziplinären Vereinnahmung entziehen, Flexibilität implizieren und verschiedenartige Themenfelder erforschen. Grob gesagt gehören zu den Leitbegriffen der Cultural Studies Pluralisierung, Identität, Macht, Anerkennung, Überlebensstrategien und Widerstandsmöglichkeiten; zu denen der Kulturwissenschaften Identität, Gender, Symbol, Medien, Gedächtnis, Ausdrucksstrategien und Lebensstile. Die Fragestellungen kennen aber keine Grenzen, wie die verschiedenen turns oder Wenden veranschaulicht haben (siehe Bachmann-Medick) oder wie es das heutige internationale Interesse für Intersektionalität oder Disability Studies (mit der Norm als Kernfrage) aufzeigt, der Heterogenitätsfaktor ist hier Relevanz, Intensität, Intentionalität. Dabei besteht das Hauptanliegen der Kulturstudien darin, Menschen in die Lage zu versetzen, ihre Weltbeziehung zu verhandeln und dabei die Normativität zu hinterfragen, die jene Beziehung prägt und beeinträchtigen mag. Dies gilt bei aller unterschiedlichen Akzentuierung; liege der Schwerpunkt auf der Gegenwart (Cultural Studies und Kulturwissenschaften) oder auf der Vergangenheit (Kulturwissenschaften), sei der Ansatz politisch (Cultural Studies) oder eher hermeneutischsemantisch (Kulturwissenschaften).

Gilt dies nun aber auch für die études culturelles, die sich als neue Forschungsformation in Frankreich auch auf das interdisziplinäre Gebiet begibt und ihre Orientierungsfunktion ausarbeitet? Das langjährige französische Potenzial an kulturwissenschaftlichem Denken (wir haben im ersten Teil von der kulturwissenschaftlichen Matrix gesprochen) hat nicht minder Keime gelegt, wie es die heutige kulturwissenschaftliche Entfaltung zeigt: Trotz einer verzögerten Rezeption ist der Einzug der Cultural Studies und Kulturwissenschaften in die französische Universitätslandschaft inzwischen unübersehbar, sei es in Form der Diskussion (siehe die Konferenzen und Seminare zum Thema Cultural Studies oder Kulturwissenschaften) oder der Adoption (siehe die Arbeiten von Georges-Claude Guilbert). Beide Entwicklungen geben zu einer wechselseitigen Befruchtung Anlass, deren Merkmale einerseits die zunehmende Ausrichtung auf Interdisziplinarität und damit Theorieüberschneidung und Methodenverflechtung sind, und andererseits die Hinwendung zu solchen Themen wie Identität, Repräsentation, Gender, Postkolonialismus, TV-Serien, Medien und neue Technologien, die in überaus dynamischen Teilen folgender Forschungsbereiche behandelt werden: Kulturgeschichte (Laurent Martin), Sozialwissenschaften (Marie-Hélène/ Sam Bourcier; Eric Macé; Eric Maigret), Komparatistik (Sébastien Hubier), Gender Studies (Geneviève Sellier), Amerikanistik (Georges-Claude Guilbert) und vor allem Kommunikationswissenschaften (Laurence Allard; Marie-Joseph Bertini; Laurent Béru; Maxime 
Cervulle; Nelly Quemener). ${ }^{3}$ Es handelt sich nicht selten um eine ästhetisch-kritisch verfahrende Kulturanalyse, die sich aber, je nach dem Forschungszusammenhang und institutionellen Rahmen, auch tendenziell im Sozialen verortet. Wie bereits gesagt, kann von keinem einheitlichen Bereich der études culturelles die Rede sein.

Zusammenfassend steht fest, dass sich Cultural Studies, Kulturwissenschaften und études culturelles zwar keine monodisziplinäre Norm der Untersuchung vorschreiben lassen, zugleich aber einer gewissen Normativität der Interdisziplinarität und wissenschaftlichen Offenheit zustimmen. Dies erklärt die Einheitlichkeit-Differenziertheit dieser kühnen Formationen, die interdisziplinäre Fragestellungen wagen, unerwartete aber doch fruchtbare Bezüge zu vergangenen und aktuellen Gegenwartsproblemen herstellen, manche Forscher von ihrer fachlichen Selbstbezüglichkeit befreien, neue kooperative Arbeitsformen initiieren und im Falle der Cultural Studies internationalen Anschluss finden. Finden sie aber dafür institutionellen Anschluss?

\section{EINHEITLICHKEIT UND DIFFERENZIERTHEIT AUF DER EBENE DER INSTITUTIONELLEN ANERKEN- NUNG UND IMPLEMENTIERUNG}

Wir wollen jetzt das Thema der Anerkennung kurz anschneiden, also der Frage nachgehen, ob Cultural Studies, Kulturwissenschaften und études culturelles zu einem normalen Element der Universitätslandschaft geworden sind, das sich in gewissem Maße in den institutionellen Rahmen eingefügt hat. Was die Kulturwissenschaften anbelangt, besetzen sie wohl ihren Platz in der deutschsprachigen Universitätslandschaft als Institute oder Forschungszentren. Damit ist aber nicht gesagt, dass ihre Legitimität unangefochten ist, wie es der unvollendete Wandel der Nomenklatur auf der Ebene der Institute und Studiengänge wie auch der Fächer in Bibliotheken, Verlagen oder der Sektionen bei wissenschaftlichen Veranstaltungen (und der Praktiken überhaupt) dokumentiert. Es gibt noch viele Fakultäten für Geisteswissenschaften; es gibt auch Studiengänge in Literatur- und Kulturwissenschaften, die nicht unbedingt interdisziplinär aufgestellt sind, wobei die Umbenennung doch eine Modernisierung der Objekte konnotiert, ohne gar eine interdisziplinäre Umstrukturierung vorauszusetzen (dabei bleibt indessen der Fokus auf der

3 Um dies anschaulich zu machen, können wir einige Titel vom Sammelband Etudes culturelles \& Cultural Studies (Darras 2007) zitieren, der die CS-Rezeption und die neuesten kulturwissenschaftlichen Entwicklungen in Frankreich thematisiert: .Etudes culturelles et minorités indisciplinées dans la France contemporaine“ (Marie-Hélène Bourcier), „La banlieue, révélatrice de l'utilité des ,French Cultural Studies'. Pour l'étude des (non-)dits ethnico-raciaux français“" (Laurent Béru), ,Les études culturelles pour penser le communautarisme en France depuis le début des années 1990" (Marie-Cécile Naves), ,Un mode nautarisme en France depuis le début des années 1990" (Marie-Cécile Naves), „Un mode
original d'appropriation des Cultural Studies. Les études de genre appliquées aux Sciences original d'appropriation des Cultural Studies. Les études de genre appliquées aux Sciences
de l'information et de la communication. Concepts, théories, méthodes et enjeux“ (Marie-
Joseph Bertini), "Les Cultural Studies et l'étude des cultures populaires“ (Paul Rasse), Joseph Bertini), ,Les Cultural Studies et l'étude des cultures populaires“ (Paul Rasse), "Culture matérielles et construction de l'identité culturelle. Discours, représentations rapports de pouvoir" (Sarah Belkhamsa \& Bernard Darras), "La circulation des contenus culturaliste“ (Laure Bolka-Tabary), „De l'articulation entre classe, race, genre et sexualite dans la pornographie 'ethnique ${ }^{x \prime}$ (Maxime Cervulle).
Textanalyse und -interpretation); es gibt auch die im Singular gebrauchte Kulturwissenschaft, die sich als Einzeldisziplin mit eigenen theoretischen und methodologischen Optionen und eigenen Gegenstandsfeldern konstituiert. Zudem stehen viele Akademiker den Gender Studies nach Art der Cultural Studies ziemlich zurückhaltend gegenüber und andere Akademiker, unter innen deutsche Vertreter der Cultural Studies, drücken schwerwiegende Vorbehalte aus (Überbewertung des Textes und der Semiologie oder Vernachlässigung vom Ökonomischen). Dabei sei festzuhalten, dass die deutsche Rezeption der Cultural Studies relativ spät (größtenteils in den neunziger Jahren) und mit ihrer Konzentration auf Medien und Jugendund Popkultur auch etwas selektiv, gar verzerrt geschieht. Es gibt also viele mögliche Wege, viele unterschiedliche interdisziplinäre Konfigurationen, die noch im Aufbau sind und sich auf den wissenschaftspolitischen Kontext (immer wieder) neu einstellen. Wie wir bereits betonten, haben die Kulturwissenschaften eine viel längere Entstehungsgeschichte als die Cultural Studies, die bis auf die Wahrnehmungs- und Denkschemata einzelner Wissenschaftler wie Lamprecht, Simmel, Benjamin, Warburg zurückreicht. Wenn der Elan durch die NS-Zeit und den Krieg abgebrochen wurde, traten doch die innovativen Konzepte der genannten Wissenschaftler in den siebziger und achtziger Jahren neu in den Blickpunkt und verschärften die Legitimationskrise der Geisteswissenschaften (angesichts der fehlenden Auseinandersetzung mit der Nazi-Vergangenheit, der wachsenden Phänomene der Hybridisierung in Folge der Globalisierung, der neuen technologischen Herausforderungen, der Mängel und Unterlassungen der Fachspezialisierung, der Impulse des postmodernistischen Denkens). Dies führte zu einer Renaissance der Kulturwissenschaften und deren Institutionalisierung an ost- und westdeutschen Universitäten in den neunziger Jahren. In diesem Zusammenhang bedeutete eine ,Umrüstung der Geistes- zu Kulturwissenschaften sowohl eine gedankliche Umorientierung als auch eine Lösung für die Umgestaltung der DDR-Universitäten wie auch für die Modernisierung der Geisteswissenschaften, die sich fortan im Kontext der Verknappung von Ressourcen vor den Steuerzahlern noch akuter rechtfertigen müssen. Mit anderen Worten sind Kulturwissenschaften zwar gleichsam normalisiert, weil institutionalisiert, erfreuen sich aber keines Schutzstatus.

In dieser Hinsicht gehen sie den gleichen Weg wie die Cultural Studies, die institutionell verankert (in Form von Instituten, Forschungszentren, Verbänden, Fachzeitschriften, usw.), aber weiterhin nicht abgesichert sind - eben, weil sie aufgrund ihres gesellschaftskritischen Impetus keine Norm erfüllen noch auferlegen wollen und weil sie aufgrund ihres Erkenntnisinteresses teilweise die Prozesshaftigkeit kulturellen Wandels, also den Wandel an sich verkörpern wollen - daher unter anderem die Kritik an der mangelnden erkenntnistheoretischen Grundlegung. ${ }^{4}$

4 Im Rahmen dieses Beitrages kann es sich nur um eine kurze, ja etwas verkürzende Bemerkung handeln. Allerdings ist die Frage der Anerkennung und Institutionalisierung de Cultural Studies so häufig behandelt worden, dass sich ein ausführliches Referat an dieser Stelle erübrigen dürfte und wir uns eher auf den Stand der études culturelles konzentrieren wollen. 
Diese Kritik gilt auch den études culturelles in Frankreich, die sich jetzt institutionell positionieren, wie etwa bei den Neugründungen von fachübergreifenden Studiengängen. Solche haben die Universitäten Montpellier 3 und Paris 1 eingerichtet: Jeweils einen Master „Etudes culturelles“, der sich auf eine entsprechende Forschungsgruppe stützt (IRIEC - Institut de Recherche Intersite d'Etudes Culturelles in Montpellier 3 und Acte-Arts, Créations, Théories et Esthétiques in Paris 1). Zu den Zugpferden der études culturelles gehören auch die Universitäten Lille 3 und Paris 3 sowie Paris 8 mit einem Master-Programm zum Thema „Gender: Denkarten der Differenz, Geschlechterbeziehungen", das durch das Centre d'études féminines et d'études de genre ${ }^{5}$ etabliert wurde. Richtungsweisend für das Kulturverständnis vieler Forscher, die sich von der Beengtheit ihrer Herkunftsdisziplinen emanzipieren wollen, sind namentlich staatlich getragene Institutionen wie die EHESS, das CNRS, die interdisziplinär angelegte Maison des Sciences de l'Homme oder auch das CIERA (Interdisziplinäres Zentrum für Studien und Forschungen über Deutschland) mit dem damaligen Forschungsprogramm "Theorien und Kritiken der Kulturwissenschaften in Europa" (2006-2008). Es liegt größtenteils an der experimentellen Rolle der französischen außeruniversitären Einrichtungen, deren Forschungszentren bzw. -gruppen nicht selten an bestimmten thematischen Schwerpunkten orientiert sind und dabei transdiziplinäre Perspektiven eröffnen. Der Durchbruch erfolgt des Weiteren über immaterielle Netzwerke, wie etwa akademische Internetforen, Websites, Newsletter und Mailinglisten, die dem Forschungspublikum Zugang zu Diskussionen und Informationen über kulturwissenschaftliche Entwicklungen und Veranstaltungen, insbesondere Call for Papers, verschaffen (wie etwa Fabula oder RECEL). Das alles sind Zeichen einer allmählichen - wiewohl zwiespältigen - Einpflanzung der études culturelles in die französische Universitätslandschaft. Dass die französische Universität ihre a-historische Autonomie zu wahren sucht und noch relativ marktunabhängig ist, hat in gewissem Maße eine graduelle, ,sanfte' kulturwissenschaftliche Entwicklung erlaubt, die durch spezifische kulturelle Herausforderungen wie ,Immigration und Integration' in letzter Zeit wohl gestützt worden ist. Aber die Finanzierungsmodalitäten der Institute und die fehlenden Ressourcen bilden Innovationsbarrieren und beeinträchtigen doch die Gestaltung neuartiger interdisziplinärer Formate. Deswegen entspricht die wachsende Aufmerksamkeit für die Cultural Studies in Frankreich keiner richtigen

5 Das für die Universitätslandschaft einzigartige Institut wurde 1974 durch Hélène Cixous gegründet.
Normalisierung. Es geht vielmehr um Prozesse der partiellen Aneignung in bestimmten Forschungsbereichen, die durch den partikularen wissenschaftlichen, wirtschaftlichen, politischen und kulturellen Zusammenhang bedingt sind. Dies sollte uns nicht wundern: Denn Kulturstudien sind radikal konjunkturell.

Dass es normale, aber kaum normierte Cultural Studies, Kulturwissenschaften und études culturelles geben kann, ist im Grunde folgerichtig: Sie sind eine mobile, flexible, reaktive Form der Auseinandersetzung mit variierenden Kontexten und somit konstitutiv heterogen. Aber ausgerechnet diese Plastizität macht gleichwohl ihre Homogenität aus: Sie verhilft nämlich der ganzen Konstellation der Forscher in den Kulturstudien zu einem kompletteren, komplexeren Verständnis des Weltgeschehens. Dabei verwerfen die Vertreter der Cultural Studies, Kulturwissenschaften und études culturelles jede Norm, die eine Standardisierung des Denkens und Verfahrens bedeuten würde und stimmen hingegen der Norm der Interdisziplinarität und des ständigen Hinterfragens zu: So können sie tatsächlich Orientierungswissen generieren, d. h. Wissen über zeitlich und räumlich Fernes und Nahes, und den Menschen Handlungskompetenzen verschaffen. Und so üben sie eine große Anziehungskraft aus und weisen ein hohes Aneignungspotenzial auf: Cultural Studies, die sich mit deutschen und französischen Kulturtheorien befass(t) en, wurden in der deutschsprachigen und etwas später in der französischen Forschung, mehr oder weniger polemisch und auch adaptiv rezipiert; Kulturwissenschaften ließen eine theoretische Dynamik in Teilen der Kulturanalyse in Frankreich entstehen, und prägten die englischsprachigen Cultural Studies durch deren ikonische Denker des 20 Jahrhunderts. Études culturelles, die nun einen Namen und einen institutionellen Status anstreben, wecken ein wachsendes Interesse bei den englisch- und deutschsprachigen Kulturstudien, indem sie eine neue kulturelle Variante der interdisziplinären Beschäftigung mit Kultur sowie eine neue Variation um die Norm zentriert darstellen. Und so lassen sie sich alle nicht so leicht institutionell etablieren, da sie jenseits der nationalen und disziplinären Grenzen und Normen denken.

Kurz gesagt, es geht ihnen nicht um Norm und Anleitungen, sondern um Orientierung und Kompetenzen. Cultural Studies, Kulturwissenschaften und études culturelles repräsentieren also unterschiedliche Formeln eines gemeinsamen Projektes der Emanzipation, die jede auf ihre (kontextuelle) Weise den Menschen intellektuelle Ressourcen verschafft, um mit der Norm bzw. den kulturellen Normen umzugehen und gegebenenfalls neue Formen des Welterlebens zu erfinden.

\section{Auswahlbibliographie}

Assmann, Aleida (2006), Einführung in die Kulturwissenschaft Grundbegriffe, Themen, Fragestellungen, Berlin, Erich Schmidt.

Bachmann-Medick, Doris (2015), Cultural Turns. Neuorientierungen in den Kulturwissenschaften, Reinbek bei Hamburg, Rowohlt.
Böhme, Hartmut / Mattussek, Peter / Müller, Lothar (Hg.) (2000), Orientierung Kulturwissenschaft. Was sie kann, was sie will, Reinbek bei Hamburg, Rowohlt.

Chalard-Fillaudeau, Anne (2015), Les études culturelles, Paris, Presses Universitaires de Vincennes. 
Darras, Bernard (dir.) (2007), Etudes culturelles \& Cultural Studies, Paris, L'Harmattan.

During, Simon (1993), The Cultural Studies Reader, London \& New York, Routledge.

Glevarec, Hervé / Macé, Eric / Maigret, Eric (dir.) (2008), Cultural Studies. Anthologie, Paris, Armand Colin.

Grossberg, Lawrence / Nelson, Cary / Treichler, Paula A. (dir.) (1992), Cultural Studies, New York, Routledge.

Grossberg, Lawrence (2010), Cultural Studies in the Future Tense, Durham and London, Duke University Press.

Guilbert, Georges-Claude (2014), Le Genre des objets, Paris, L'Harmattan.

Hall, Stuart (1992), Culture, Media, Language: Working Papers in Cultural Studies, 1972-79, London, Routledge.

Horak, Roman (2002), Die Praxis der Cultural Studies, Wien, Löcker.
Kaenel, André / Lejeune, Catherine / Rossignol, Marie-Jeanne (dir.) (2003), Cultural Studies. Etudes Culturelles, Nancy, Presses Universitaires de Nancy.

Maderthaner, Wolfgang / Musner, Lutz (2010), L'autoliquidation de la raison. Les sciences de la culture et la crise du social, Übersetzung und Einleitung: Chalard-Fillaudeau, Anne, Paris, Editions de la Maison des sciences de l'homme.

Mattelart, Armand / Neveu, Erik (2003), Introduction aux Cultural Studies, Paris, La Découverte.

Musner, Lutz / Wunberg, Gotthart (Hg.) (2003), Kulturwissenschaften. Forschung - Praxis - Positionen, Freiburg im Breisgau, Rombach Verlag. Musner, Lutz (1999), „Locating culture in the US and central Europe a transatlantic perspective on cultural studies“, Cultural Studies, Vol. 13, Issue 4, Routledge.

Nünning, Ansgar (2005), Grundbegriffe der Kulturtheorie und Kulturwissenschaften, Stuttgart, J.B. Metzler. 